\title{
Effects of $\gamma$-synuclein on the tumorigenicity and metastasis of colon cancer SW1116 cells in vitro and in vivo
}

\author{
QING YE ${ }^{1-3}$, FENG HUANG $^{1-3}$, XIAO-YING WANG ${ }^{2,3}$, YANG-MEI XU ${ }^{2,3}$, FU-SHENG GONG ${ }^{2,3}$, \\ LI-JIE HUANG ${ }^{2,3}$, CHUN-KANG YANG ${ }^{1-3}$, QIU-HONG ZHENG ${ }^{2,3}$ and MIN-GANG YING ${ }^{1-3}$ \\ ${ }^{1}$ Department of Abdominal Surgery, and ${ }^{2}$ Tumor Surgery Research Office, \\ Teaching Hospital of Fujian Medical University, Fujian Provincial Tumor Hospital; \\ ${ }^{3}$ Fujian Key Laboratory of Tumor Biological Treatment, Fuzhou, Fujian 350014, P.R. China
}

Received July 2, 2013; Accepted August 2, 2013

DOI: $10.3892 / o r .2013 .2688$

\begin{abstract}
Recent evidence suggests the involvement of $\gamma$-synuclein in tumorigenesis and tumor progression. The present study was designed to further clarify the effects of $\gamma$-synuclein on the biological features of colon cancer cells in vitro and in vivo. We constructed the eukaryotic expression vector and siRNA vector and selected stable transfectants to respectively upregulate and downregulate $\gamma$-synuclein expression in SW1116 cells. We found that silencing of $\gamma$-synuclein significantly attenuated SW1116 cell growth and colony formation in vitro $(\mathrm{P}<0.05)$, and overexpression of $\gamma$-synuclein moderately enhanced cell growth and colony formation, but not significantly when compared with the parental SW1116 cells and empty vector-transfected cells $(\mathrm{P}>0.05)$. Meanwhile, overexpression of $\gamma$-synuclein significantly facilitated SW1116 cell migration, invasion and adhesion to human liver sinusoidal endothelial cells (HLSECs) in vitro $(\mathrm{P}<0.05)$, and the effects were less attenuated by $\gamma$-synuclein knockdown $(\mathrm{P}>0.05)$. Furthermore, $\gamma$-synuclein promoted these malignant phenotypes in a $\gamma$-synuclein expression quantity-dependent manner not only in vitro but also in the in vivo expression. Stable cells were injected subcutaneously into the right flank, and injected intrasplenically in nude mice. $\gamma$-synuclein knockdown suppressed the tumorigenicity of SW1116 cells in mice, which presented significantly smaller tumor masses on day 6 over a 30-day period, compared with empty vector cells $(\mathrm{P}<0.05)$. Meanwhile, overexpression of $\gamma$-synuclein led to a profound augmentation of liver metastasis in nude mice, not only in macroscopic appearance but also in the size and weight of livers $(\mathrm{P}<0.05)$. These results provide strong evidence that suggests $\gamma$-synuclein plays a positive role in the progression of colorectal cancer.
\end{abstract}

Correspondence to: Dr Feng Huang or Professor Min-Gang Ying, Department of Abdominal Surgery, Fujian Provincial Tumor Hospital, 420 Fuma Road, Fuzhou, Fujian 350014, P.R. China E-mail: huangfeng19731@163.com

E-mail: m_g_ying@163.com

Key words: $\gamma$-synuclein, colorectal cancer, tumorigenicity, metastasis, in vitro, in vivo

\section{Introduction}

Colorectal cancer (CRC) is one of the most frequently diagnosed malignancies in both men and women worldwide, and CRC is the second most common cause of cancer-related mortality in developed countries such as the USA (1). Traditional and combined therapeutic approaches (surgery, chemotherapy and radiation) for curing $\mathrm{CRC}$ give rise to improvements in progression-free and overall survival, yet, the outcome of this common malignancy remains unsatisfactory mainly due to metastasis or recurrent disease, poor understanding of the mechanism of CRC development and the lack of specific target gene therapy $(2,3)$. In recent years, multiple studies have been conducted to investigate the genes and their products that are involved in the progression of CRC (4-6).

The synucleins $(\alpha-, \beta$ - and $\gamma$-synuclein) are a small, soluble, highly conserved group of neuronal proteins that have attracted considerable attention due to their involvement in neurodegenerative disorders such as Alzheimer's and Parkinson's disease. Synuclein expression is commonly highly tissue-specific and is restricted to brain tissue and presynaptic terminals $(7,8)$. However, aberrant expression of synucleins beyond the neuronal system has been highly associated with human malignancies. The $\gamma$-synuclein gene was initially cloned from infiltrating breast carcinoma cells by using the expressed sequence tag-based differential cDNA sequencing approach (9), and stage-specific expression of $\gamma$-synuclein has been found in advanced breast cancers and other malignancies, including ovarian, pancreatic and bladder cancers (10-13). The $\alpha$-synuclein gene was identified in patients with bladder cancer by oligonucleotide arrays, and $\alpha$-synuclein protein is significantly associated with tumor staging and clinical outcome (14). $\alpha$ - and $\beta$-synucleins have also been found to be expressed in several nervous system cancers and breast and ovarian cancer $(15,16)$.

In our previous study, we investigated expression patterns of synucleins in CRC tissues and cell lines, and found that among $\alpha$-, $\beta$ - and $\gamma$-synucleins, $\gamma$-synuclein was most significantly correlated with CRC (17). We also provided clinical evidence suggesting that $\gamma$-synuclein expression is upregulated in $\mathrm{CRC}$, which is primarily attributed to the demethylation of the $\mathrm{CpG}$ island in exon 1 , and that aberrant expression 
Table I. Sequences of $\gamma$-synuclein gene-specific primers and shRNA.

\begin{tabular}{ll}
\hline Oligonucleotides & \multicolumn{1}{c}{ Sequence (5'-3') } \\
\hline$\gamma$-synuclein-5' (RT-PCR) & CAAGAAGGGCTTCTCCATCGCCAAGG \\
$\gamma$-synuclein-3' (RT-PCR) & CCTCTTTCTCTTTGGATGCCACACCC \\
GAPDH-5' & GAAGGTGAAGGTCGGAGTC \\
GAPDH-3' & GAAGATGGTGATGGGATTTC \\
$\gamma$-synuclein-5' (full-length PCR) & CCCTCGAGGGATGGATGTCTTCAAGAAGG \\
$\gamma$-synuclein-3' (full-length PCR) & CGGGATCCCGCTAGTCTCCCCCACTCG \\
$\gamma$-synuclein-5' (shRNA) & GATCCGGAGAATGTTGTACAGAGCTTCAAGAGAGCTCTGTACAACATTCTCCTTTTTTGGAAA \\
$\gamma$-synuclein-5' (shRNA) & AGCTTTTCCAAAAAAGGAGAATGTTGTACAGAGCTCTCTTGAAGCTCTGTACAACATTCTCCG
\end{tabular}

and demethylation of $\gamma$-synuclein are closely correlated with advanced clinical stage, lymph node involvement and distant metastasis (18). Experimental data from previous studies have also revealed a tumor phenotype of $\gamma$-synuclein in other types of cancer. Overexpression of $\gamma$-synuclein was found to lead to a significant increase in motility and invasiveness in breast cancer cell culture and to a profound enhancement in metastasis in nude mice $(19,20) . \gamma$-synuclein has been shown to compromise normal mitotic checkpoint controls, resulting in multinucleation as well as more rapid breast cancer cell growth $(21,22) \cdot \gamma$-synuclein has been implicated in late stage ovarian cancer metastasis by enhancing cell motility through activation of the RHO family small-GTPases and ERK1/2 (23).

Collectively, these findings suggest a potential role of $\gamma$-synuclein in the process of tumorigenesis and metastasis. However, little is known concerning the biological effects of $\gamma$-synuclein in CRC cell line culture and in animal models. In the present study, we investigated the effects of $\gamma$-synuclein on the biological features of colon cancer cell line SW1116 by cell growth curve, soft agar assay, cell migration, invasion and adhesion assays in vitro, and by a tumor xenograft model of tumorigenicity and a liver metastasis assay in vivo.

\section{Materials and methods}

Cell cultures. The colon cancer cell line SW1116 (ATCC no. CCL-233) was grown in RPMI-1640 medium (Gibco-BRL, Life Technologies Inc., Gaithersburg, MD, USA) supplemented with $10 \%$ heat inactivated fetal bovine serum (FBS) (Summit Biotechnology, Fort Collins, CO, USA). The colon cancer cell line HT-29 (ATCC no. HTB-38) was cultured in McCoy's 5a medium (Gibco-BRL) supplemented with $10 \%$ FBS. Human liver sinusoidal endothelial cells (HLSECs) were purchased from Cell Systems (Kirkland, WA, USA) and grown in Dulbecco's modified Eagle's medium (DMEM) (Gibco-BRL) supplemented with $10 \%$ FBS. Human umbilical vein endothelial cells (HUVECs) (ATCC no. PCS-100-013) were obtained from ATCC and maintained in F12K supplemented with endothelial cell growth supplement (ECGS) $(0.05 \mathrm{mg} / \mathrm{ml})$ (BD Biosciences, Bedford, MA, USA) and heparin (Sigma-Aldrich, Victoria, Australia) $(0.1 \mathrm{mg} / \mathrm{ml})$. Cells were maintained in a humidified incubator at $37^{\circ} \mathrm{C}$ with $5 \% \mathrm{CO}_{2}$, fed every 3 days with complete medium and subcultured when confluence was reached.

$R T$-PCR. Total RNA was extracted using TRIzol reagent (Invitrogen, Carlsbad, CA, USA). Synthesis of cDNA from $1 \mu \mathrm{g}$ RNA was performed with a reverse transcription system kit (Promega, Madison, WI, USA). PCR reactions were carried out in a thermal cycler (model PTC-225; M J Research, Watertown, MA, USA) using HotStarTaq DNA polymerase (Qiagen $\mathrm{GmbH}$, Hilden, Germany) as follows: $95^{\circ} \mathrm{C}$ for $15 \mathrm{~min}, 34$ cycles of $94^{\circ} \mathrm{C}$ for $30 \mathrm{sec}, 60^{\circ} \mathrm{C}$ for $30 \mathrm{sec}$, and $72^{\circ} \mathrm{C}$ for $30 \mathrm{sec}$, and finally at $72^{\circ} \mathrm{C}$ for $5 \mathrm{~min}$. The expression of GAPDH mRNA was taken as an internal loading control. The PCR products were separated on $1.5 \%$ agarose gels and stained with ethidium bromide. Table I provides the sequences of the primers used in the study.

Plasmid construction. Total RNA was extracted from HT29 cells, and the full-length cDNA of $\gamma$-synuclein was amplified using RT-PCR. The digested fragment of cDNA was inserted between the XhoI and BamHI sites of the pIRES2-EGFP plasmid to generate the pIRES2- $\gamma$-synuclein construct. The sequences (as shown in Table I) unique to the coding region of $\gamma$-synuclein were chemically synthesized and inserted between the BamHI and HindIII sites of the pGCsi-U6/neo/ GFP plasmid to generate the pGCsi- $\gamma$-synuclein construct. The positive clones of pIRES2- $\gamma$-synuclein and pGCsi- $\gamma$-synuclein were confirmed by sequencing.

Plasmid transfection and selection of the SW1116 stable transfectants. One day before transfection, SW1116 cells were plated in a 6 -well plate at $1 \times 10^{5}$ cells/well using RPMI-1640 medium without antibiotics. The cells were transfected with $3.0 \mu \mathrm{g} /$ well of the recombinant plasmids and empty plasmids as control, respectively, using Lipofectamine (Invitrogen). Fresh growth medium was replaced after $4 \mathrm{~h}$ of transfection. Cells were passaged at a 1:10 dilution at $24 \mathrm{~h}$ after transfection and cultured in medium supplemented with G418 (Promega) at $1,000 \mu \mathrm{g} / \mathrm{ml}$ for 4 weeks. Stably transfected polyclones were selected from several cell islands with green fluorescent protein (GFP or EGFP) and were maintained in medium containing $400 \mu \mathrm{g} / \mathrm{ml} \mathrm{G} 418$ for further study. Meanwhile, GFP or EGFP fluorescence was detected by flow cytometry to confirm that the purity of transfectants was $>95 \%$. 
Western blot analysis. Cells were harvested and lysed with mammalian protein extraction reagent (Pierce, Rockford, IL, USA). The lysated proteins were quantified by bicinchoninic acid (BCA) protein assay kit (Pierce). Total protein $(50 \mu \mathrm{g})$ was subjected to $15 \%$ SDS-PAGE and electrophoretically transferred to PVDF membranes (Millipore, Bedford, MA, USA). Membranes were blocked overnight with 5\% skimmed milk in TBS buffer containing $0.05 \%$ Tween- 20 at $4^{\circ} \mathrm{C}$, and incubated with a 1:500 dilution of mouse anti- $\gamma$-synuclein monoclonal antibody (sc-65979; Santa Cruz Biotechnology, Santa Cruz, CA, USA) and goat anti-mouse IgG-AP antibody (Santa Cruz Biotechnology) (1:5,000), respectively. Membranes incubated with ECL (Pierce) for 1 min were exposed to the film for 1-5 min. GAPDH served as a loading control.

Cell proliferation analysis. Cells were plated in five copies onto 96 -well plates at a density of $2 \times 10^{3}$ cells/well in $100 \mu \mathrm{l}$ RPMI-1640 medium containing $10 \% \mathrm{FBS}$ at $37^{\circ} \mathrm{C}$ with $5 \%$ $\mathrm{CO}_{2}$. The number of viable cells was determined daily with the WST- 8 cytotoxicity assay using the Cell Counting Kit- 8 (Dojindo, Japan). Briefly, $10 \mu 1$ of the CCK- 8 solution was added to each well of the microplate, and the absorbance at $490 \mathrm{~nm}$ was measured by a microplate reader ( $\mu$ Quant; Bio-Tek, Winooski, VT, USA) after a 4-h incubation.

Soft agar colony formation assay. Cells $\left(1 \times 10^{3}\right)$ were trypsinized to a single-cell suspension and then plated in triplicate onto 6-well plates in complete culture medium containing $0.3 \%$ agar on top of $0.6 \%$ agar in the same medium. Cultures were maintained at $37^{\circ} \mathrm{C}$ with $5 \% \mathrm{CO}_{2}$ incubator for 14 days. The colonies were fixed with $70 \%$ ethanol and stained with $0.2 \%$ crystal violet. The colonies containing at least 50 cells were counted. Colony formation rates were calculated as the number of colonies relative to that of cells initially plated in a well $\left(1 \times 10^{3}\right)$, and were expressed as mean \pm SD.

Wound healing assay. Cells were plated in 35-mm dishes to form a monolayer one day before assay. After making a straight scratch with a pipette tip, cells were incubated in RPMI-1640 medium containing $10 \% \mathrm{FBS}$ at $37^{\circ} \mathrm{C}$ with $5 \% \mathrm{CO}_{2}$ for $36 \mathrm{~h}$. The motile cells were photographed under light microscopy to detect the speed of wound closure at various intervals. Assays were repeated three times.

Cell invasion assay. Boyden chambers with $8-\mu \mathrm{m}$ polycarbonate membranes in 24-well dishes (Nucleopore, Pleasanton, CA, USA) were coated with $4 \mathrm{mg} / \mathrm{ml}$ growth factor-reduced Matrigel (50 $\mu \mathrm{g}$; Collaborative Biomedical, Becton Dickinson

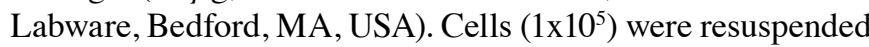
in serum-free RPMI-1640 and added to the upper chamber in triplicate. Consecutively, RPMI-1640 with 10\% FBS was added to the lower chamber. Chambers were incubated at $37^{\circ} \mathrm{C}$ with $5 \% \mathrm{CO}_{2}$ incubator. After a 48-h incubation, the chambers were fixed with $70 \%$ ethanol, and cells were stained with $0.2 \%$ crystal violet. Cells on the surface of the upper chamber were removed by swiping with cotton swabs. The number of invasive cells in the lower chamber was determined under light microscopy. The data are expressed as means \pm SD of cell counts in 10 random fields of vision.
Cell adhesion assay. For the adhesion assay with HLSECs or HUVECs, a 96-well plate, coated by $2 \%$ gelatin, was seeded with $1 \times 10^{4}$ endothelial cells/well and cultured until confluent. SW1116 cells $\left(5 \times 10^{4}\right)$ were then transferred to each well in triplicate and left to adhere to the endothelial layer for $1 \mathrm{~h}$ at $37^{\circ} \mathrm{C}$. The endothelial layer was washed with PBS to remove the non-adherent cells. Since $\gamma$-synuclein expression plasmids contained green fluorescence protein (GFP or EGFP), the attached cells were quantified by measuring the fluorescence intensity using a microplate reader (model Safire 2; Tecan, Männedorf, Switzerland).

Tumor xenograft model of tumorigenicity and liver metastasis assay. Six-week-old male BALB/c nu/nu nude mice were purchased from the Experimental Animal Centre of Shanghai Institutes for Biological Sciences (SIBS, Shanghai, China) and housed in a specific pathogen-free environment. The pIRES2-EGFP, pIRES2- $\gamma$-synuclein, pGCsi-U6/ neo/GFP, and pGCsi- $\gamma$-synuclein cells were harvested, washed and re-suspended in PBS, respectively. For the tumorigenicity assay, $1 \times 10^{6}$ cells suspended in $100 \mu \mathrm{l}$ PBS were injected subcutaneously into the right flank regions using a 27-gauge needle. Mice were checked every 3 days for tumor appearance, and tumor size was determined by measuring two diameters with a caliper. Tumor volume (V) was estimated by using the equation: $V=4 / 3 \pi \times L / 2 \times$ $(\mathrm{W} / 2)^{2}$, where $\mathrm{L}$ is the mid-axis length and $\mathrm{W}$ is the mid-axis width. For liver metastasis assay, mice were anesthetized intraperitoneally with $2.5 \%$ Avertin (Sigma). The spleen was exteriorized through a left lateral flank incision, and $1 \times 10^{6}$ cells in $50 \mu \mathrm{l}$ of PBS were injected into the distal tip of spleen parenchyma using a 27 -gauge needle. The injection site on the spleen was pressed with a cotton stick wet in iodine-polividone solution in order to destroy extravasated cells and ensure hemostasis. The peritoneum and skin were closed in a single layer with surgical thread. Each group for the tumorigenicity assay included 10 mice and each group for the liver metastasis assay included 7 mice. All animals were sacrificed on day 30 after tumor cell implantation. Tumor specimens (in the right flank, liver and spleen) were collected, fixed in $10 \%$ neutral buffered formalin, embedded in paraffin and subjected to hematoxylin and eosin (H\&E) staining and immunohistochemistry (IHC). The Ethics Committee of the Fujian Medical University approved the animal usage in the research, and all surgical procedures and care administered to the animals were in accordance with institutional guidelines.

Histology and immunohistochemistry. Unstained 4-mm sections were cut from tissue blocks and stained with $\mathrm{H} \& \mathrm{E}$ and subjected to IHC analysis. Sections subjected to IHC were cleared with xylene and rehydrated with ethanol, and the slides were bathed in $0.01 \mathrm{~mol} / 1$ sodium citrate and heated in a microwave oven for $12 \mathrm{~min}$. The sections were incubated with anti- $\gamma$-synuclein antibody (sc-65979; Santa Cruz Biotechnology) and kept at $4^{\circ} \mathrm{C}$ overnight. Negative control slides were treated only with non-immunized mouse immunoglobulin fraction under equivalent conditions. For the secondary developing reagents, a labeled streptavidin-biotin kit (Dako, Carpinteria, CA, USA) was used. Slides were 
$\mathbf{A}$
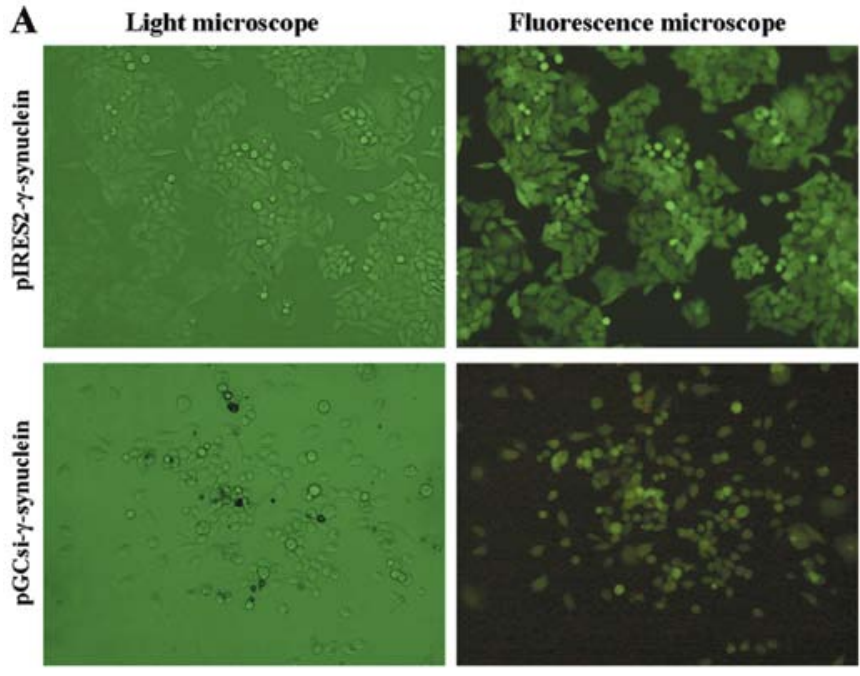

B

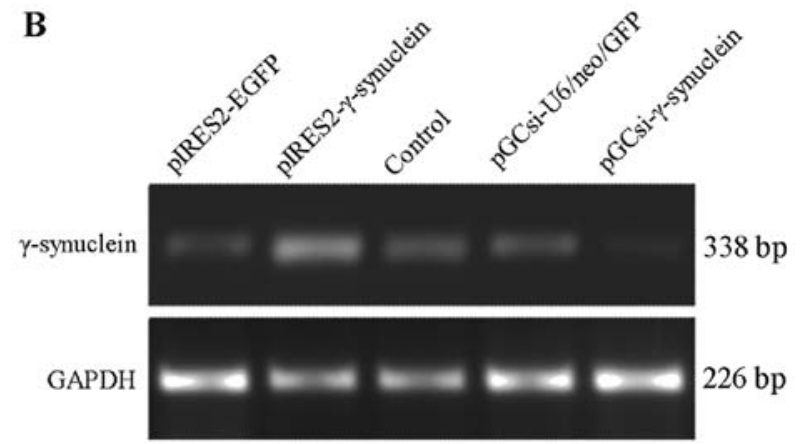

C

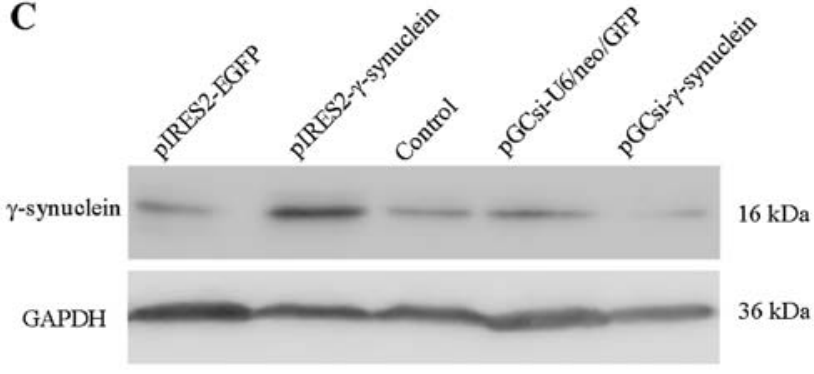

Figure 1. Identification of SW1116 stable transfectants. (A) Images of the SW1116 stable transfectants using fluorescence and light microscopy. (B) RT-PCR analysis of $\gamma$-synuclein mRNA in parental SW1116 (control) and stable transfectants. (C) Western blot analysis of $\gamma$-synuclein protein in control and stable transfectants.

developed with diaminobenzaminidine and counterstained with hematoxylin.

Statistical analysis. The results are presented as means \pm SD. Differences between groups were compared by two-way ANOVA, and were considered significant at $\mathrm{P}<0.05$. Data analysis was carried out using SSPS 13.0 software.

\section{Results}

Identification of SW1116 stable transfectants. After identification by sequence analysis, $\gamma$-synuclein gene eukaryotic expression plasmids and siRNA plasmids were successfully constructed. The recombinant plasmids and empty plasmids were transfected into SW1116 cells. After 4 weeks of selection
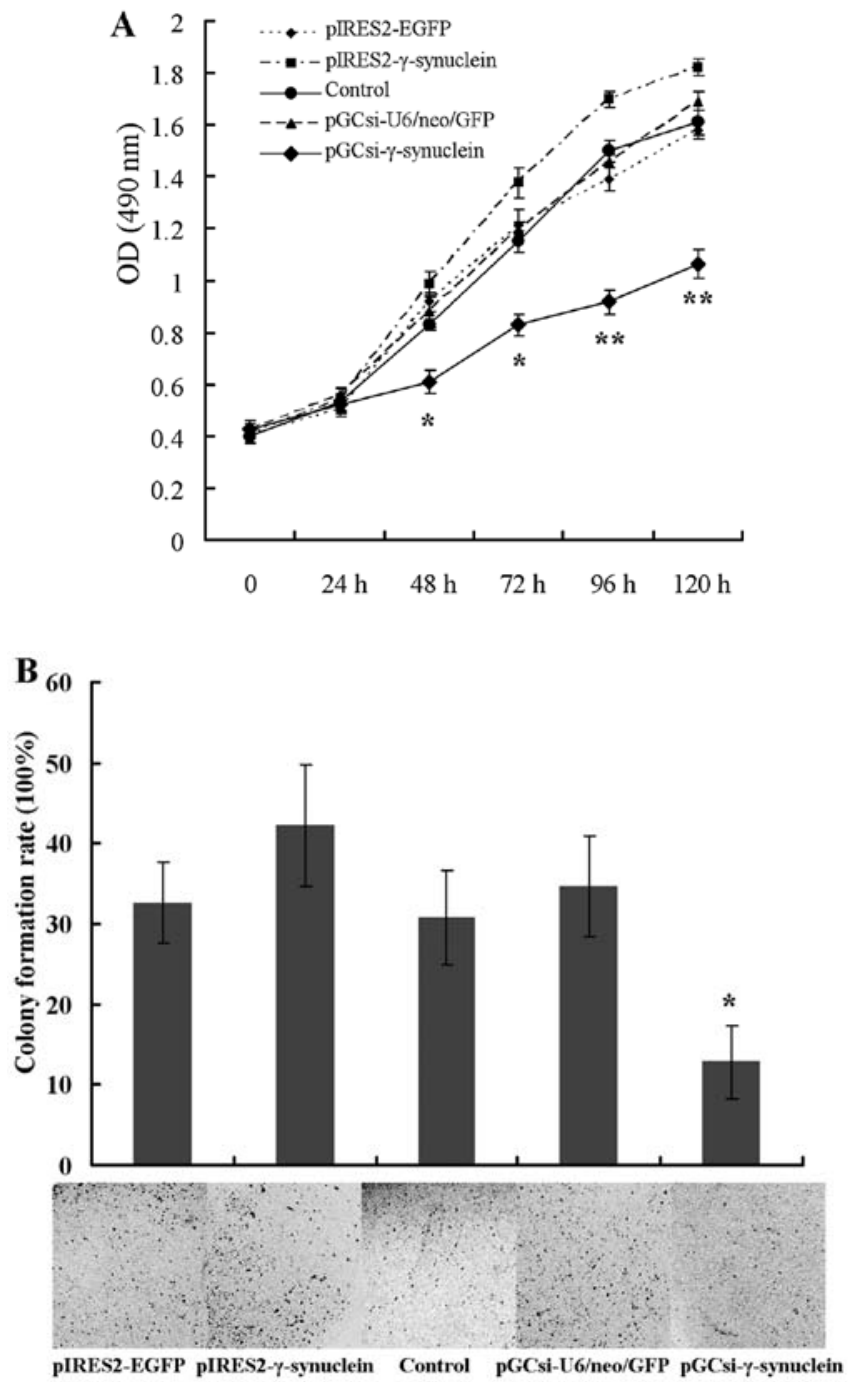

Figure 2. Effects of $\gamma$-synuclein on cell proliferation and colony formation. (A) The role of $\gamma$-synuclein in regulating SW1116 cell proliferation was determined by the CCK- 8 assay. Values are the mean \pm SD of absorbance at $490 \mathrm{~nm}$ for five independent experiments; $\mathrm{P}<0.05$, ${ }^{* *} \mathrm{P}<0.01$. (B) The colony formation rates were analyzed by soft agar assay. Values are the mean $\pm \mathrm{SD}$ for three independent experiments, ${ }^{*} \mathrm{P}<0.05$.

with G418, stable transfected clones were successfully established, and the purity of the transfectants was $>95 \%$ (Fig. 1A). To confirm the $\gamma$-synuclein expression in the stable transfectants, we examined the expression of $\gamma$-synuclein by RT-PCR and western blot analysis. The expression of $\gamma$-synuclein mRNA was upregulated in pooled pIRES2- $\gamma$-synuclein cells and downregulated in pooled pGCsi- $\gamma$-synuclein cells (Fig. 1B); these finding paralleled those of the western blot analysis (Fig. 1C).

Effects of $\gamma$-synuclein on SW1116 cell proliferation and colony formation in vitro. In vitro cell proliferation assay showed that $\gamma$-synuclein knockdown significantly suppressed cell growth, and the number of pGCsi- $\gamma$-synuclein cells was significantly reduced by $48,72,96$ and $120 \mathrm{~h}$ after plating, respectively, compared with the corresponding empty plasmid cells and parental (control) cells (Fig. 2A; P<0.05). The number of pIRES2- $\gamma$-synuclein cells was increased, but not significantly (Fig. 2A; P>0.05), compared with the control 


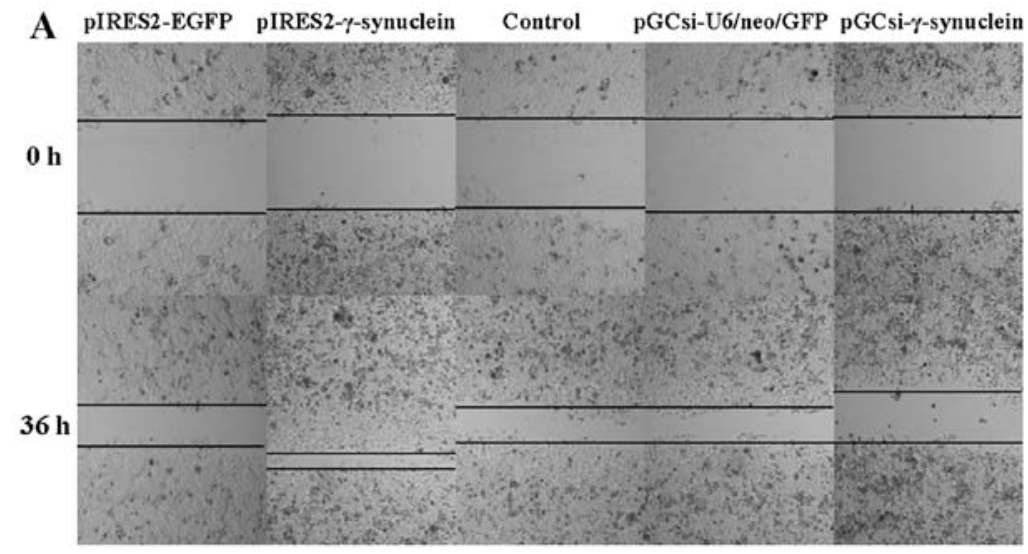

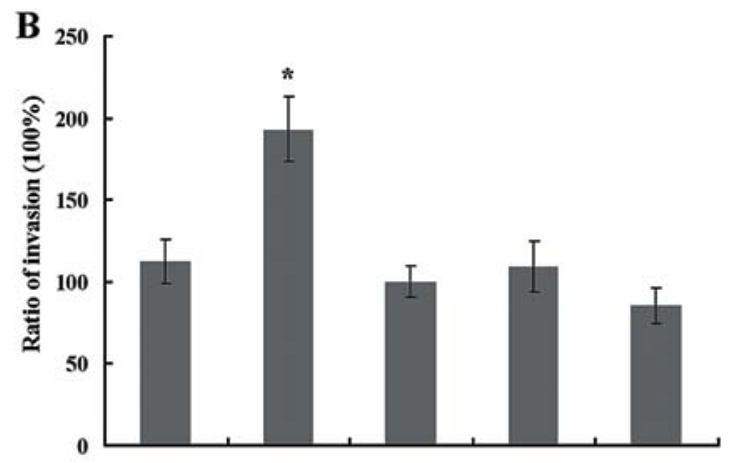

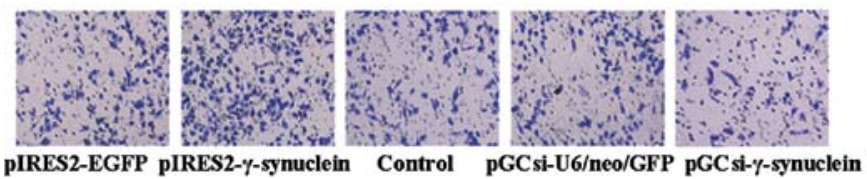

pIRES2-EGFP pIRES2-\%-synuclein Control pGCsi-U6/neo/GFP pGCsi- --synuclein

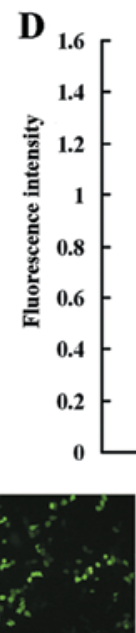

pIRES2-EGFP

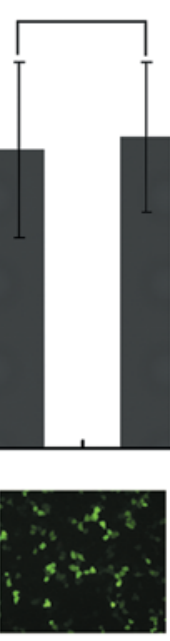

pIRES2- $\gamma$-synuclein

\section{HUVECs}

Figure 3. Effects of $\gamma$-synuclein on SW1116 cell migration, invasion and adhesion. (A) Representative images of migrated cells. The solid lines indicate the areas occupied by the initial wound or healing wound. (B) The number of invasive cells was counted in 10 random fields of vision. Values are the number of cells relative to that of the parental SW1116 cells (control), and are expressed as the mean $\pm \mathrm{SD}$ of three independent experiments; ${ }^{*} \mathrm{P}<0.05$. (C and D) Representative images of SW1116 cell adhesion to (C) HLSECs or (D) HUVECs. The attached cells were quantified by measuring the fluorescence intensity of EGFP or GFP by a microplate reader, and values are expressed as the mean $\pm \mathrm{SD}$ of three independent experiments; ${ }^{*} \mathrm{P}<0.05$.

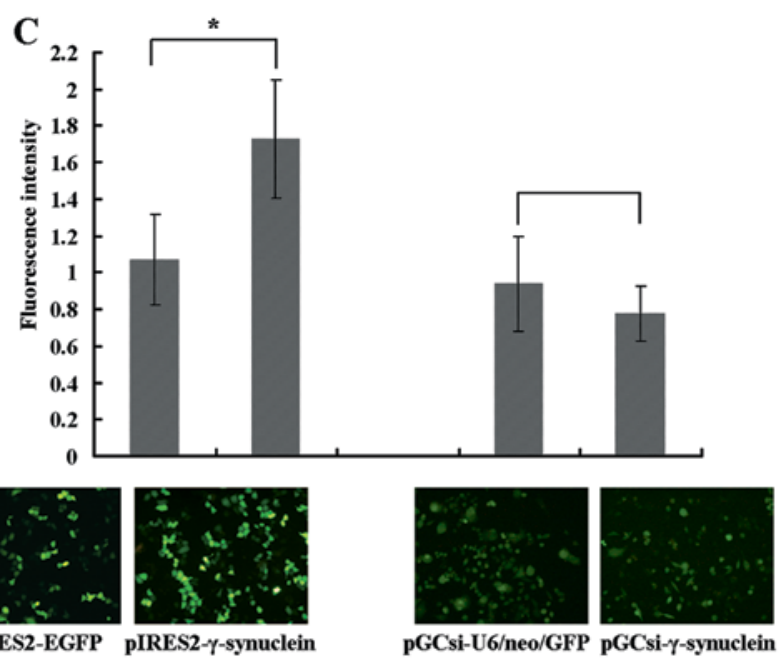

HLSECs

and pIRES2-EGFP cells, as well as the pGCsi-U6/neo/GFP and pGCsi- $\gamma$-synuclein cells, which in general presented the effects in a $\gamma$-synuclein expression quantity-dependent manner. Subsequent soft agar colony formation assay was conducted to evaluate the tumorigenicity of cells in vitro. Colony formation rates were $32.6 \pm .1,42.2 \pm 7.5,30.8 \pm 5.9,34.2 \pm 6.2$ and $12.8 \pm 4.6 \%$ in the pIRES2-EGFP, pIRES2- $\gamma$-synuclein, control, pGCsi-U6/neo/GFP and pGCsi- $\gamma$-synuclein cells. The colony formation rate of the pGCsi- $\gamma$-synuclein cells was significantly reduced, compared with the empty vector and control cells (Fig. 2B; P<0.05). The size of colonies formed by the pGCsi$\gamma$-synuclein cells was smaller than that of the two control cells. Whereas in parallel with the results of the proliferation assay, overexpression of $\gamma$-synuclein moderately enhanced colony formation of the SW1116 cells (Fig. 2B; P>0.05), which also exhibited a $\gamma$-synuclein expression quantity-dependent trend.

Effects of $\gamma$-synuclein on SW1116 cell migration, invasion and adhesion in vitro. The results of the wound healing assay demonstrated that $\gamma$-synuclein is closely involved in cell motility. SW1116 cells overexpressing $\gamma$-synuclein moved more rapidly towards the gap, compared with the empty vector and control cells, and $\gamma$-synuclein knockdown decreased the healing rate of the SW1116 cell injury (Fig. 3A). A subsequent reconstituted basement membrane (Matrigel) invasion assay 

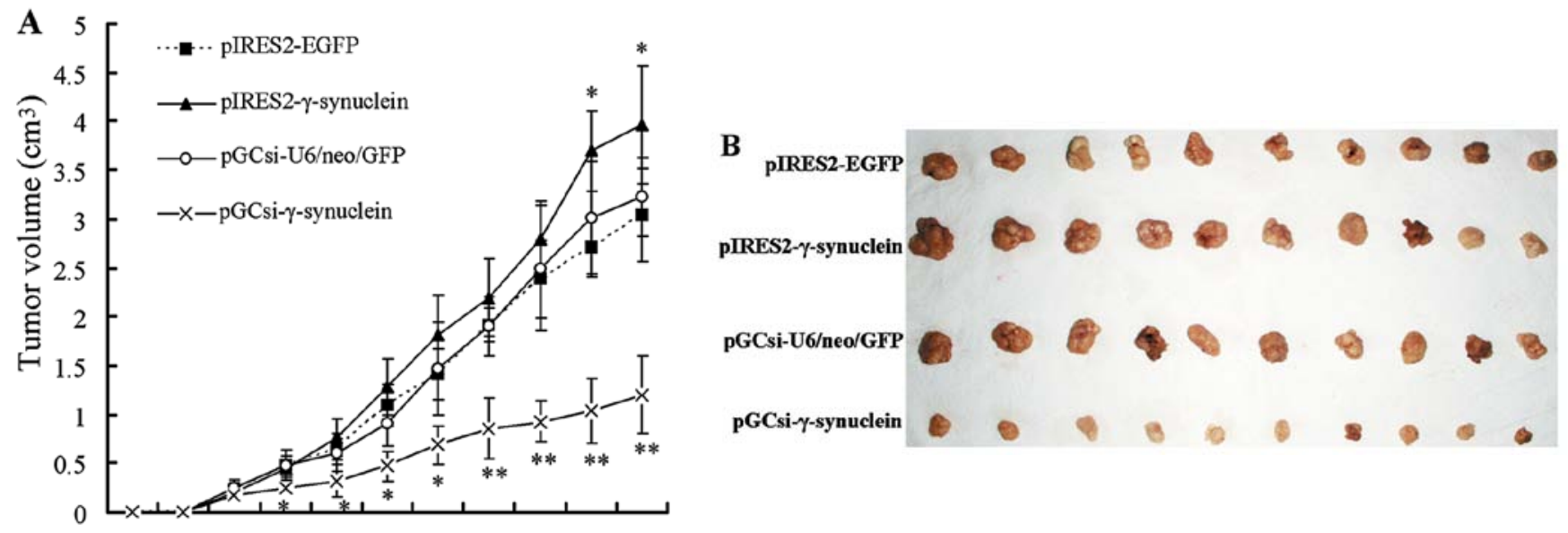

$0 \quad 3 \mathrm{~d} \quad 6 \mathrm{~d} \quad 9 \mathrm{~d} 12 \mathrm{~d} 15 \mathrm{~d} 18 \mathrm{~d} 21 \mathrm{~d} 24 \mathrm{~d} 27 \mathrm{~d} 30 \mathrm{~d}$

C
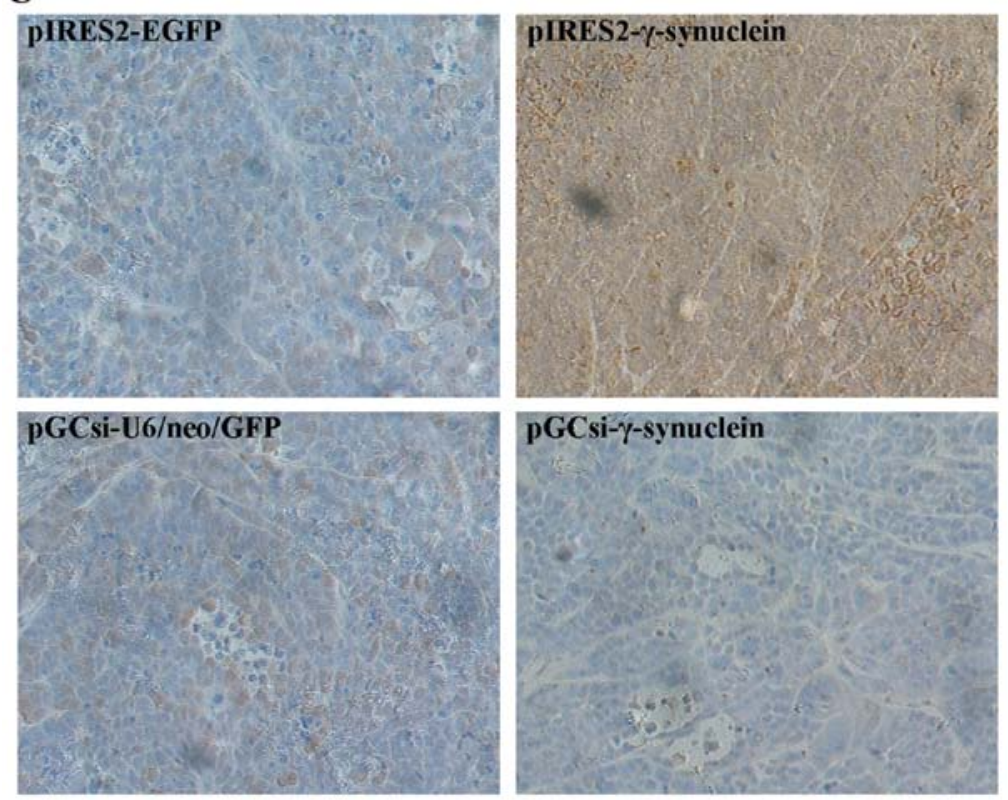

Figure 4. Effects of $\gamma$-synuclein on SW1116 cell tumorigenicity in vivo. (A) Tumor dimensions were measured every 3 days over a 30-day period. Each point represents the average volume (mean $\pm \mathrm{SD}$ ) calculated from 10 mice; ${ }^{*} \mathrm{P}<0.05,{ }^{* *} \mathrm{P}<0.01$. (B) Images of tumor specimens from the injected mice on day 30 . (C) Images of IHC analysis of $\gamma$-synuclein protein expression in tumors (original magnification, $\mathrm{x} 200$ ).

was carried out. As expected, $\gamma$-synuclein facilitated SW1116 cell invasion through Matrigel and a filter membrane, and the number of invasive pIRES2- $\gamma$-synuclein cells was increased by 93.1 and $81.5 \%$, respectively; a higher number than that of the control and empty vector cells (Fig. 3B; $\mathrm{P}<0.05$ ). However, $\gamma$-synuclein knockdown did not significantly attenuate invasion of SW1116 cells, compared with the two corresponding control cells (Fig. 3B; P>0.05). Generally speaking, $\gamma$-synuclein promoted SW1116 cell migration and invasion in a $\gamma$-synuclein expression quantity-dependent trend, which was observed in the following in vivo assays.

We carried out adhesion assays to investigate whether $\gamma$-synuclein influences the adhesion of SW1116 cells to endothelial cells. In the HLSEC adhesion assay, the average fluorescence intensity of EGFP was $1.07 \pm 0.25$ in the pIRES2EGFP cells and $1.73 \pm 0.32$ in the pIRES2- $\gamma$-synuclein cells; $\gamma$-synuclein significantly facilitated SW1116 cell adhesion to HLSECs (Fig. 3C; $\mathrm{P}<0.05$ ). There was no difference in fluorescence intensity of GFP between the pGCsi-U6/neo/GFP $(0.94 \pm 0.26)$ and pGCsi- $\gamma$-synuclein $(0.78 \pm 0.15)$ cells (Fig. 3C; $\mathrm{P}>0.05)$. Meanwhile, the results of the HUVEC adhesion assay revealed no effect of $\gamma$-synuclein on the adhesive ability of SW1116 cells to HUVECs (Fig. 3D; P>0.05), which suggests that SW1116 cells overexpressing $\gamma$-synuclein interact specifically with HLSECs.

Effects of $\gamma$-synuclein on SW1116 cell tumorigenicity in vivo. We examined the in vivo tumorigenicity of SW1116 cells by injecting pIRES2-EGFP, pIRES2- $\gamma$-synuclein, pGCsi-U6/neo/ GFP and pGCsi- $\gamma$-synuclein cells subcutaneously into the right flank regions of nude mice. The xenograft tumor growth rates in the different groups were compared. Xenograft tumors appeared in all mice of the three groups except for the pGCsi$\gamma$-synuclein group on day 6 , and pIRES2- $\gamma$-synuclein group mice exhibited a faster tumor growth rate from day 27 to the end of the expreriment, compared to the other three groups 
Table II. Intraperitoneal dissemination nodules of the tumors.

No. of nodules

\begin{tabular}{lcccc} 
Transfectants & Tumor incidence $(\%)$ & Mesentery & Gastrointestinal serosa & Peritoneum \\
\hline pIRES2-EGFP & $4 / 7(57.1)$ & 11 & 5 & 3 \\
pIRES2- $\gamma$-synuclein & $7 / 7(100)$ & 28 & 8 & 4 \\
pGCsi-U6/neo/GFP & $3 / 7(42.9)$ & 8 & 3 & 4 \\
pGCsi- $\gamma$-synuclein & $2 / 7(28.6)$ & 6 & 2 & 1 \\
\hline
\end{tabular}

(Fig. 4A; $\mathrm{P}<0.05$ ). However, only 6 of the 10 mice injected with pGCsi- $\gamma$-synuclein cells presented with tumors on day 6 , and the other 4 mice remained with no tumor formation until day 9. All pGCsi- $\gamma$-synuclein cell tumors maintained a slow rate of growth, compared to the other three groups, within the time remaining until sacrifice (Fig. 4A; $\mathrm{P}<0.05$ ). All animals were sacrificed on day 30 and tumor tissue specimens were collected as shown in Fig. 4B. The expression of $\gamma$-synuclein in tumor tissues was detected by IHC staining. The results showed that $\gamma$-synuclein protein was upregulated in tumors derived from pIRES2- $\gamma$-synuclein transfectants and downregulated in tumors derived from pGCsi- $\gamma$-synuclein transfectant, when compared with the two empty vector transfectants. (Fig. 4C).

Effects of $\gamma$-synuclein on SW1116 cell liver metastasis in vivo. We adopted an experimental model that entailed the injection of tumor cells into the spleen of nude mice, followed by assessment of their ability for tumorigenesis in the spleens and invasion via the portal vein into the liver and potential intraperitoneal dissemination. All animals were sacrificed by laparotomy on day 30, and tumor progression was observed macroscopically. All mice developed primary tumors in the spleen, and all mice injected with pIRES2- $\gamma$-synuclein cells developed metastases on the liver surface. Meanwhile, mice infected with pIRES2- $\gamma$-synuclein developed multiple intraperitoneal dissemination nodules in the mesentery, gastrointestinal serosa and peritoneum, whease less tumor nodules were found in the peritoneal cavity of mice in the other three groups (Table II). Liver and spleen specimens were collected for further evaluation. Liver replacement by metastases was too extensive in the liver of $71.4 \%$ ( $n=5$ of 7 ) of pIRES2- $\gamma$ synuclein mice, which displayed enlarged livers covered with numerous tumor nodules throughout and with a whitish and irregular surface caused by extensive tumor growth, whereas the livers of the other groups were smaller in size, displaying a macroscopically normal appearance with a few surface nodules, and no liver surface metastases were found in 2 of 7 pIRES2-EGFP, 3 of 7 pGCsi-U6/neo/GFP and 3 of 7 pGCsi$\gamma$-synuclein mice (Fig. 5A). The results paralleled the weight of the livers (Fig. 5B), which also exhibited a $\gamma$-synuclein expression quantity-dependent trend; the more $\gamma$-synuclein protein expressed, the more obvious the liver metastasis. As shown in Fig. 5C, the growth of these cells in the spleens was almost identical in the pIRES2- $\gamma$-synuclein and two empty vector groups, and was significantly decreased in the pGCsi- $\gamma$ synuclein group $(\mathrm{P}<0.05)$. Liver specimens were subsequently subjected to histologic examination by $\mathrm{H} \& \mathrm{E}$ staining
(Fig. 5D). The results showed that liver metastases were found microscopically in all mice including micrometastases in mice without macroscopic nodules. Large regions of liver tissues were replaced by metastases with formation of neovessels, which was reported to be required for tumors to grow beyond a critical size, whereas livers containing pGCsi- $\gamma$-synuclein cells were mainly occupied by small-sized metastases with central necrosis.

\section{Discussion}

Colon carcinogenesis and metastasis is a multistage process involving alterations of various tumor-suppressor genes and oncogenes $(24,25)$. In recent years, multiple studies have been conducted to investigate the genes and proteins correlated with progression of $\mathrm{CRC}$; however, no internationally recognized molecular marker has been identifed for potential clinical implications (4-6). $\gamma$-synuclein is a promising biomarker, belonging to the synuclein protein family, which was initially investigated in the field of neurodegenerative diseases $(7,8)$. Although one study showed downregulation of $\gamma$-synuclein in human esophageal squamous cell carcinoma (26), additional studies including ours support the overexpression and the oncological role of $\gamma$-synuclein in a variety of cancers $(10-13,18)$. The prognostic impact of $\gamma$-synuclein overexpression is impressive, and it was found to be the only independent predictor of reduced overall survival and the strongest negative indicator of disease-free survival by multivariate analysis in breast cancer (10).

On the basis of our previous study on expression and demethylation of $\gamma$-synuclein in CRC tissues and cell lines $(17,18)$, we constructed the $\gamma$-synuclein gene eukaryotic expression and siRNA vectors, and established permanent transfected SW1116 cells to investigate the biological functions of $\gamma$-synuclein. Our data showed that silencing of $\gamma$-synuclein in SW1116 cells contributed to suppression of cellular proliferation and colony formation in vitro. These results were consistent with observations of the $\gamma$-synuclein siRNA vector in HCT116 cells in our previous study and as well as in other reports of breast and ovarian cancer $(21-23,27)$. The overexpression of $\gamma$-synuclein moderately facilitated proliferation and colony formation of SW1116 cells, but not significantly compared with the other groups of cells, which presented a promotive effect in a $\gamma$-synuclein expression quantity-dependent manner. Similar findings were also found in the tumorigenicity assay in vivo. In the in vivo tumorigenicity assay, $\gamma$-synuclein-knockdown SW1116 cells formed significantly smaller tumor masses than 

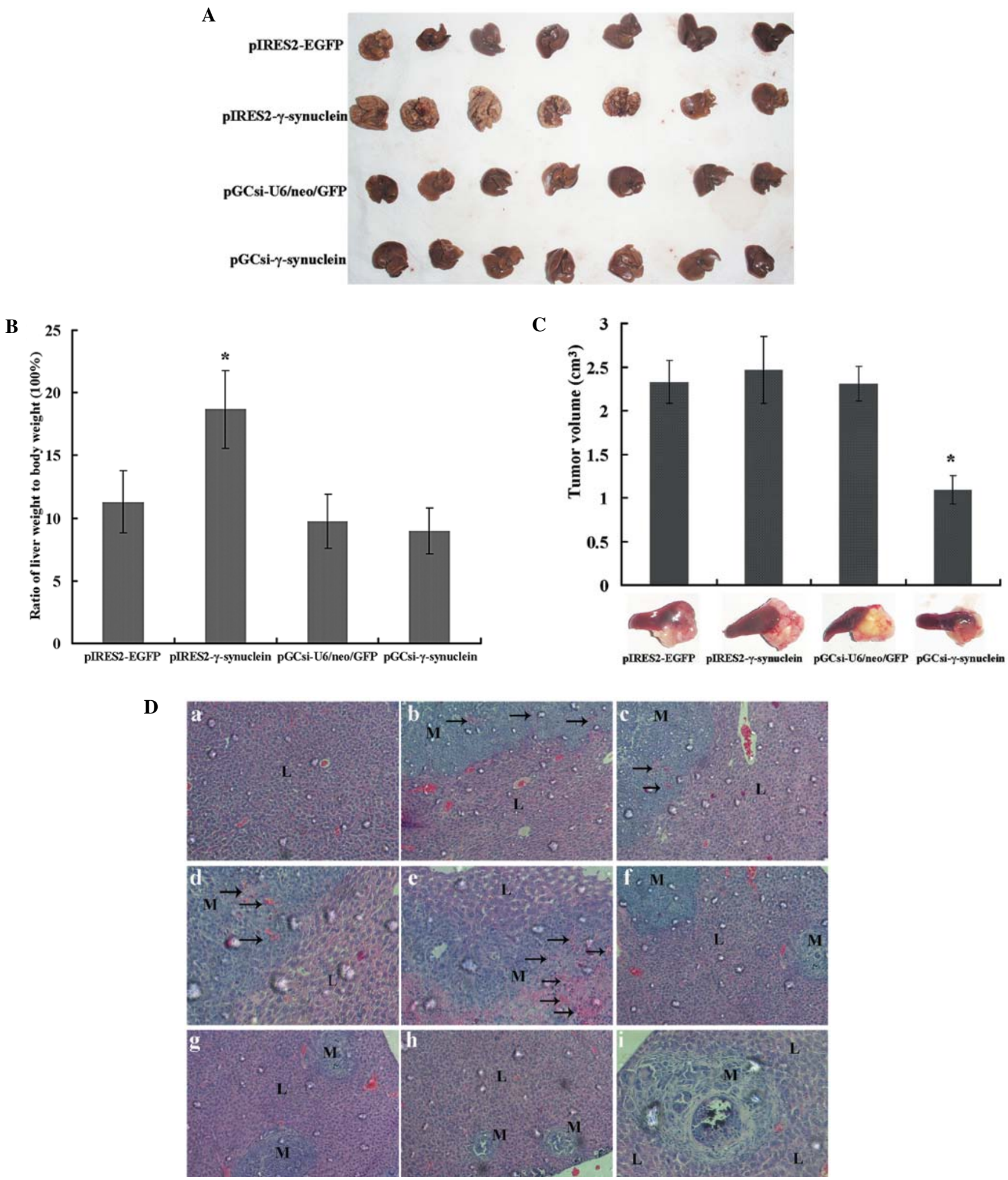

Figure 5. Effects of $\gamma$-synuclein on SW1116 cell liver metastasis in vivo. (A) Macroscopic images of liver metastases at 30 days after intrasplenic injection of tumor cells. (B) Quantitative analysis of the weights of tumor-bearing livers on day 30 and expressed as the mean $\pm \mathrm{SD}$ from 7 mice; " $\mathrm{P}<0.05$. (C) Representative images of primary tumors in the spleen. The tumor volume was determined on day 30 and expressed as mean \pm SD from 7 mice; " $P<0.05$. (D) Representative photomicrographs of H\&E-stained sections of liver tissues from mice following intrasplenic injection of tumor cells (original magnification, a-c, f-h, x200; d, e, i, x400). (a) Normal liver tissues of pGCsi- $\gamma$-synuclein mice. (b-e) Liver tissues from pIRES2- $\gamma$-synuclein mice, which presented large regions of liver metastases with formation of neovessels. (f) Liver metastases from pIRES2-EGFP mice. (g) Liver metastases from pGCsi-U6/neo/GFP mice. (h and i) Liver tissues from pGCsi- $\gamma$-synuclein mice, which presented small-sized metastases with central necrosis. L, liver; M, metastases; arrows, neovessels.

the empty vector cells on day 6 over a 30 -day period, whereas tumors derived from overexpressed $\gamma$-synuclein cells did not significantly exhibit faster tumor growth until day 27 . The probable reasons are as follow. Upregulation of $\gamma$-synuclein 
frequently contributes to cancer cell survival but not proliferation, and moderate expression of $\gamma$-synuclein is enough for cell proliferation through an HSP- or ER-based multiprotein chaperone complex. However, overexpression of $\gamma$-synuclein may cause occupation of all binding sites in the multiprotein chaperone complex, with no site left for remaining $\gamma$-synuclein proteins $(10,28,29)$.

Metastasis leads to $90 \%$ of CRC-related mortality, yet, the underlying mechanisms remain largely unclear (30). Metastasis is a sequential process that includes detachment from the primary site, invasion and adhesion to vasculature, translocation through the systemic circulation, extravasation into the parenchyma of distant tissues (liver and lungs) and colonization of a distant site (31). In order to model this process, we therefore, developed migration, invasion and adhesion assays in vitro, and a liver metastasis assay in vivo. The results of these assays also exhibited a $\gamma$-synuclein expression quantity-dependent trend; the more $\gamma$-synuclein protein expressed, the more obvious was the promotion of these malignant phenotypes. In vitro, upregulation of $\gamma$-synuclein promoted SW1116 cell migration through a Matrigel and a filter membrane, and adherence of SW1116 cells to HLSECs but not HUVECs. Similar effects of $\gamma$-synuclein on cell migration and invasion have been reported in breast and ovarian cancer, as well as effects on adhesion as in the report of $\mathrm{Hu}$ et al who found that SW1116 subpopulation, SW1116p21, with high potential for liver metastasis that highly express $\gamma$-synuclein, exhibited enhanced adhesion to HLSECs $(19,23,32)$, whereas downregulation of $\gamma$-synuclein did not significantly inhibit SW1116 cell ability for migration, invasion and adhesion to HLSECs. Our previous experimental data revealed that downregulation of $\gamma$-synuclein significantly inhibited the migration and invasion ability of HCT116 cells. The discordant results may be due to the difference in metastatic features between HCT116 and SW1116 cells, and the effects of $\gamma$-synuclein siRNA vector were not apparent in relative weak metastatic SW1116 cells. The similar magnitude of migration and invasion-stimulating activity, and special interaction with HLSECs suggests that $\gamma$-synuclein enhances the SW1116 cell capacity for invasion and metastasis, which were later confirmed by an experimental model of liver metastasis in vivo. Overexpression of $\gamma$-synuclein facilitated SW1116 cell liver metastases, and apparent differences were found not only in macroscopic appearance but also in size and the weight of livers, which further confirmed the positive role of $\gamma$-synuclein in the invasion and liver metastasis potential of colon cancer cells. Livers containing pGCsi- $\gamma$-synuclein cells had less and small-sized metastases with central necrosis, consistent with observations of the effects of $\gamma$-synuclein siRNA on cell proliferation and colony formation. It is likely that survival and proliferation in systemic circulation and colonization at a distant site are also important steps in the sequential process of metastasis. On the other hand, except for the pGCsi- $\gamma$-synuclein group, the growth rate of tumors in the spleens was almost the same, whereas the intraperitoneal dissemination tumor rate was different, which collectively suggests that liver metastases occurred independent of a direct effect on primary tumor growth in the study.

In summary, we used in vitro and in vivo assays to study the effects of $\gamma$-synuclein on tumorigenicity and metastasis of the colon cancer cell line SW1116. Through construction of $\gamma$-synuclein gene eukaryotic expression and siRNA vectors, and selection of SW1116 stable transfectants, respectively, we found that downregulation of $\gamma$-synuclein inhibited the proliferation and colony formation of SW1116 cells in vitro and tumorigenicity in nude mice. Meanwhile, upregulation of $\gamma$-synuclein enhanced SW1116 cell ability of migration, invasion and adhesion to HLSECs in vitro and liver metastasis in nude mice. Furthermore, $\gamma$-synuclein promoted these malignant phenotypes of SW1116 cells in a $\gamma$-synuclein expression quantity-dependent manner not only in vitro but also in vivo. This is also the first report of the in vivo evidence of $\gamma$-synuclein effects on a colon cancer cell line, which further suggests that $\gamma$-synuclein plays a positive role in the progression of CRC, and may serve as a novel molecular target for potential clinical application.

\section{Acknowledgements}

The present study was supported by the National Natural Science Foundation of China (81101897) and the Science Foundation of Fujian Province, China (2011J05061).

\section{References}

1. Siegel R, Naishadham D and Jemal A: Cancer statistics. CA Cancer J Clin 63: 11-30, 2013.

2. Mayo SC and Pawlik TM: Current management of colorectal hepatic metastasis. Expert Rev Gastroenterol Hepatol 3: 131-144, 2009.

3. Berri RN and Abdalla EK: Curable metastatic colorectal cancer: recommended paradigms. Curr Oncol Rep 11: 200-208, 2009.

4. Hartwell KA, Muir B, Reinhardt F, Carpenter AE, Sgroi DC and Weinberg RA: The Spemann organizer gene, Goosecoid, promotes tumor metastasis. Proc Natl Acad Sci USA 103: 18969-18974, 2006.

5. Mani SA, Yang J, Brooks M, et al: Mesenchyme Forkhead 1 (FOXC2) plays a key role in metastasis and is associated with aggressive basal-like breast cancers. Proc Natl Acad Sci USA 104: 10069-10074, 2007.

6. Yang J, Mani SA, Donaher JL, et al: Twist, a master regulator of morphogenesis, plays an essential role in tumor metastasis. Cell 117: 927-939, 2004.

7. Surguchov A: Molecular and cellular biology of synucleins. Int Rev Cell Mol Biol 270: 225-317, 2008.

8. Ahmad M, Attoub S, Singh MN, Martin FL and El-Agnaf OM: $\gamma$-Synuclein and the progression of cancer. FASEB J 21 : 3419-3430, 2007.

9. Ji H, Liu YE, Jia T, et al: Identification of a breast cancer-specific gene, BCSG1, by direct differential cDNA sequencing. Cancer Res 57: 759-764, 1997.

10. Guo J, Shou C, Meng L, et al: Neuronal protein synuclein $\gamma$ predicts poor clinical outcome in breast cancer. Int J Cancer 121: 1296-1305, 2007.

11. Hibi T, Mori T, Fukuma M, et al: Synuclein- $\gamma$ is closely involved in perineural invasion and distant metastasis in mouse models and is a novel prognostic factor in pancreatic cancer. Clin Cancer Res 15: 2864-2871, 2009.

12. Dokun OY, Florl AR, Seifert HH, Wolff I and Schulz WA: Relationship of SNCG, S100A4, S100A9 and LCN2 gene expression and DNA methylation in bladder cancer. Int J Cancer 123: 2798-2807, 2008.

13. Liu H, Liu W, Wu Y, et al: Loss of epigenetic control of synuclein- $\gamma$ gene as a molecular indicator of metastasis in a wide range of human cancers. Cancer Res 65: 7635-7643, 2005.

14. Sanchez-Carbayo M, Socci ND, Lozano J, Saint F and Cordon-Cardo C: Defining molecular profiles of poor outcome in patients with invasive bladder cancer using oligonucleotide microarrays. J Clin Oncol 24: 778-789, 2006.

15. Fung KM, Rorke LB, Giasson B, Lee VM and Trojanowski JQ: Expression of alpha-, beta-, and gamma-synuclein in glial tumors and medulloblastomas. Acta Neuropathol 106: 167-175, 2003. 
16. Bruening W, Giasson BI, Klein-Szanto AJ, Lee VM, Trojanowski JQ and Godwin AK: Synucleins are expressed in the majority of breast and ovarian carcinomas and in preneoplastic lesions of the ovary. Cancer 88: 2154-2163, 2000.

17. Ye Q, Wang TF, Peng YF, et al: Expression of $\alpha-, \beta$ - and $\gamma$-synuclein in colorectal cancer, and potential clinical significance in progression of the disease. Oncol Rep 23: 429-436, 2010.

18. Ye Q, Zheng MH, Cai Q, et al: Aberrant expression and demethylation of $\gamma$-synuclein in colorectal cancer, correlated with progression of the disease. Cancer Sci 99: 1924-1932, 2008.

19. Jia T, Liu YE, Liu J and Shi YE: Stimulation of breast cancer invasion and metastasis by synuclein $\gamma$. Cancer Res 59: 742-747, 1999.

20. Surgucheva IG, Sivak JM, Fini ME, Palazzo RE and Surguchov AP: Effect of $\gamma$-synuclein overexpression on matrix metalloproteinases in retinoblastoma Y79 cells. Arch Biochem Biophys 410: 167-176, 2003.

21. Gupta A, Inaba S, Wong OK, Fang G and Liu J: Breast cancerspecific gene 1 interacts with the mitotic checkpoint kinase BubR1. Oncogene 22: 7593-7599, 2003.

22. Inaba S, Li C, Shi YE, Song DQ, Jiang JD and Liu J: Synuclein gamma inhibits the mitotic checkpoint function and promotes chromosomal instability of breast cancer cells. Breast Cancer Res Treat 94: 25-35, 2005.

23. Pan ZZ, Bruening W and Godwin AK: Involvement of RHO GTPases and ERK in synuclein- $\gamma$ enhanced cancer cell motility. Int J Oncol 29: 1201-1205, 2006.

24. Gennari L, Russo A and Rossetti C: Colorectal cancer: what has changed in diagnosis and treatment over the last 50 years? Tumori 93: 235-241, 2007.
25. Samoha S and Arber N: Cyclooxygenase-2 inhibition prevents colorectal cancer: from the bench to the bed side. Oncology 69: 33-37, 2005.

26. Zhou CQ, Liu S, Xue LY, et al: Down-regulation of $\gamma$-synuclein in human esophageal squamous cell carcinoma. World J Gastroenterol 9: 1900-1903, 2003.

27. Pan ZZ, Bruening W, Giasson BI, Lee VM and Godwin AK: $\gamma$-synuclein promotes cancer cell survival and inhibits stress- and chemotherapy drug-induced apoptosis by modulating MAPK pathways. J Biol Chem 277: 35050-35060, 2002.

28. Liu YE, Pu W, Jiang Y, Shi D, Dackour R and Shi YE: Chaperoning of estrogen receptor and induction of mammary gland proliferation by neuronal protein synuclein gamma. Oncogene 26: 2115-2125, 2007.

29. Jiang Y, Liu YE, Goldberg ID and Shi YE: $\gamma$-synuclein, a novel heat-shock protein-associated chaperone, stimulates ligand-dependent estrogen receptor $\alpha$ signaling and mammary tumorigenesis. Cancer Res 64: 4539-4546, 2004.

30. Gupta GP and Massague J: Cancer metastasis: building a framework. Cell 127: 679-695, 2006.

31. Fidler IJ: The pathogenesis of cancer metastasis: the 'seed and soil' hypothesis revisited. Nat Rev Cancer 3: 453-458, 2003.

32. Hu H, Sun L, Guo C, et al: Tumor cell-microenvironment interaction models coupled with clinical validation reveal CCL2 and SNCG as two predictors of colorectal cancer hepatic metastasis. Clin Cancer Res 15: 5485-5493, 2009. 